International Mathematical Forum, Vol. 9, 2014, no. 14, 689 - 693 HIKARI Ltd, www.m-hikari.com http://dx.doi.org/10.12988/imf.2014.39184

\title{
On a Reversed Maruster-type Inequality and Strong Convergence of the Mann Iterative Sequence for Demicontractive Maps in Hilbert Spaces
}

\author{
B. G. Akuchu \\ Department of Mathematics \\ University of Nigeria \\ Nsukka, Nigeria
}

Copyright (c) 2014 B. G. Akuchu. This is an open access article distributed under the Creative Commons Attribution License, which permits unrestricted use, distribution, and reproduction in any medium, provided the original work is properly cited.

\begin{abstract}
Let $H$ be a real Hilbert space and $C$ be a nonempty closed convex subset of $H$. Let $T: C \rightarrow C$ be a demicontractive map satisfying $\langle x-T x, p\rangle \geq$ 0 , for all $(x, p) \in C \times F(T)$, where $F(T)=\{x \in C: T x=x\}$. Then the Mann iterative sequence given by $x_{n+1}=\left(1-\alpha_{n}\right) x_{n}+\alpha_{n} T x_{n}$, where $\alpha_{n} \in(0,1) \forall n \geq 0$, converges strongly to an element of $F(T):=\{x \in$ $C: T x=x\}$.
\end{abstract}

\section{Introduction}

Let $E$ be a real Hilbert space. A mapping $T: H \rightarrow H$ is said to be demicontractive if there exists a constant $k>0$ such that

$$
\|T x-p\|^{2} \leq\|x-p\|^{2}+k\|x-T x\|^{2}
$$

for all $(x, p) \in H \times F(T)$, where $F(T):=\{x \in H: T x=x\} \neq \emptyset$. More often than not, $K$ is assumed to be in the interval $(0,1)$. However, this is a restriction of convenience. If $k=1$, then $T$ is called a hemicontractive map. On the otherhand, $T$ is said to satisfy condition $(A)$ if there exists $\lambda>0$ such that

$$
\langle x-T x, x-p\rangle \geq \lambda\|x-T x\|^{2}
$$


for all $(x, p) \in H \times F(T)$.

The above classes of maps were studied independently by Hicks and Kubicek [3] and Maruster [5]. It is however shown in [1] that the two classes of maps coincide if $k \in(0,1)$ and $\lambda \in\left(0, \frac{1}{2}\right)$.

The class of demicontractive maps includes the class of quasi-nonexpansive and the class of strictly pseudocontractive maps. Any strictly pseudocontractive mapping with a nonempty fixed point set is demicontractive.

If $C$ is a closed convex subset of any Banach space $E$ and $T: C \rightarrow C$ is any map, then the Mann iteration sequence [2] is given by $x_{n+1}=\left(1-\alpha_{n}\right) x_{n}+$ $\alpha_{n} T x_{n}$, where $\alpha_{n} \in(0,1) \forall n \geq 0$, satisfying certain conditions. Several authors (see e.g [2], [3], [4], [5], [6]) have studied the convergence of the Mann iteration sequence to fixed points of certain mappings in certain Banach spaces. However, the Mann iteration sequence [2] is very suitable for the study of convergence to fixed points of demicontractive mappings. It is well known (see e.g [1]) that demicontractivity alone is not sufficient for the convergence of the Mann iteration sequence. Some additional smoothness properties of $T$ such as continuity and demiclosedness are necessary.

A map $T$ is said to be demiclosed at a point $x_{0}$ if whenever $\left\{x_{n}\right\}$ is a sequence in the domain of $T$ such that $\left\{x_{n}\right\}$ converges weakly to $y_{0} \in D(T)$ and $\left\{T x_{n}\right\}$ converges strongly to $x_{0}$, then $T y_{0}=x_{0}$.

In [7], Maruster studied the convergence of the Mann iteration sequence for demicontractive maps, in finite dimensional spaces, with an application to the study of the so-called relaxation algorithm for the solution of a particular convex feasibility problem. More precisely, he proved the following:

Theorem 1 [7]. Let $T: \Re^{m} \rightarrow \Re^{m}$ be a nonlinear mapping, where $\Re^{m}$ is the $m$-Euclidean space. Suppose the following are satisfied:

(i) $I-T$ is demiclosed at 0

(ii) $T$ is demicontractive with constant $k$, or equivalently $T$ satisfies condition $A$ with $\lambda=\frac{1-k}{2}$. Then the Mann iteration sequence converges to a point of $F(T)$ for any starting $x_{0}$.

Maruster [1] noted that in infinite dimensional spaces, demicontractivity and demiclosedness of $T$ are not sufficient for strong convergence. However, the two conditions ensure weak convergence. More precisely, he proved the following:

Theorem 2 [5] Let $T: C \rightarrow C$ be a nonlinear mapping with $F(T) \neq \emptyset$, where $C$ is a closed convex subset of a real Hilbert space $H$. Suppose the following conditions are satisfied:

(i) $I-T$ is demiclosed at 0

(ii) $T$ is demicontractive with constant $k$, or equivalently $T$ satisfies condition $A$ with $\lambda=\frac{1-k}{2}$

(iii) $0<a \leq \alpha_{n} \leq b<2 \lambda=1-k$

Then the Mann iteration sequence converges weakly to a fixed point of $F(T)$, for any starting $x_{0}$. 


\section{Strong Convergence}

As noted above, demicontractivity and demiclosedness of $T$ are not sufficient for strong convergence of the Mann iteration sequence in infinite dimensional spaces. Some additional conditions on $T$, or some modifications of the Mann iteration sequence are required for strong convergence to fixed points of demicontractive maps. Such additional conditions or modifications have been studied by several authors (see e.g [3], [4], [5], [6], [8], [9])

There is however an interesting connection between the strong convergence of the Mann iteration sequence to a fixed point of a demicontractive map, $T$, and the existence of a non-zero solution of a certain variational inequality. This connection was observed by Maruster [], and has been studied by several authors. More precisely, Maruster proved the following theorem:

Theorem 3 [5] Suppose $T$ satisfies the conditions of theorem 2. If in addition there exists $0 \neq h \in H$ such that $\langle x-T x, h\rangle \leq 0$ for all $x \in D(T)$, then starting from a suitable $x_{0}$, the Mann iteration sequence converges strongly to an element of $F(T)$.

The conditions of/and the variational inequality in theorem 3 have been used and generalized by several authors (see e.g [8], [9]). The existence of a non-zero solution to the variational inequality is sometimes gotten under very stringent conditions. In [1] remark 4, Maruster and Maruster made the following observation "It would therefore be interesting to study more closely the existence of a non-zero solution of the variational inequality".

The purpose of this paper is to provide a mild condition, with a reversed Maruster - type inequality, that $T$ satisfies for the strong convergence of the Mann iteration sequence to a fixed point of a demicontractive map. The condition is embodied in the following theorem:

Theorem 4 Suppose $T$ satisfies:

(i) The conditions of theorem 2

(ii) $\langle x-T x, p\rangle \geq 0$ for all $(x, p) \in C \times F(T)$. Then starting from a suitable $x_{0}$, the Mann iteration sequence converges strongly to an element of $F(T)$.

Proof From the proof of theorem 2 (see e.g [5]), we have $\left\|x_{n+1}-p\right\|^{2} \leq$ $\left\|x_{n}-p\right\|^{2}-\alpha_{n}\left(1-\alpha_{n}-k\right)\left\|x_{n}-T x_{n}\right\|^{2}$. This yields, from condition (iii) of theorem 2 , that

$$
\left\|x_{n+1}-p\right\|^{2} \leq\left\|x_{n}-p\right\|^{2}
$$

Let $0 \neq p \in F(T)$. Choose $x_{0} \in C$ such that $\langle p, p\rangle \geq\left\langle x_{0}, p\right\rangle$. This implies $\left\langle p-x_{0}, p\right\rangle \geq 0$. Then there exists $\epsilon_{0}>0$ such that $\left\langle p-x_{0}, p\right\rangle \geq \epsilon_{0}\left\|x_{0}-p\right\|^{2}$. 
Assume $\left\langle p-x_{n}, p\right\rangle \geq \epsilon_{0}\left\|x_{n}-p\right\|^{2}$. Then

$$
\begin{aligned}
\left\langle p-x_{n+1}, p\right\rangle & =\left\langle p-\left[\left(1-\alpha_{n}\right) x_{n}+\alpha_{n} T x_{n}\right], p\right\rangle \\
& =\left\langle p-x_{n}+\alpha_{n}\left(x_{n}-T x_{n}\right), p\right\rangle \\
& =\left\langle p-x_{n}, p\right\rangle+\alpha_{n}\left\langle x_{n}-T x_{n}, p\right\rangle \\
& \geq\left\langle p-x_{n}, p\right\rangle \text { since }\langle x-T x, p\rangle \geq 0 \forall(x, p) \in C \times F(T) \\
& \geq \epsilon_{0}\left\|x_{n}-p\right\|^{2} \\
& \geq \epsilon_{0}\left\|x_{n+1}-p\right\|^{2} \operatorname{from}(1.3)
\end{aligned}
$$

Hence for all $n \geq 0$, we have $\epsilon_{0}\left\|x_{n}-p\right\|^{2} \leq\left\langle p-x_{n}, p\right\rangle$ and since $x_{n} \rightarrow p$ by theorem 2 , then we have $x_{n} \rightarrow p$.

Remark The initial choice of $x_{0}$ can be done in several ways. For example if we fix $\alpha \in(0,1)$, we can choose $x_{0}=\alpha p$. With these, we have

$$
\begin{aligned}
\left\langle p-x_{0}, p\right\rangle & =\langle p-\alpha p, p\rangle \\
& =(1-\alpha)\|p\|^{2} \\
& >(1-\alpha)^{3}\|p\|^{2} \\
& \geq(1-\alpha)\|(1-\alpha) p\|^{2} \\
& =\epsilon_{0}\left\|x_{0}-p\right\|^{2}
\end{aligned}
$$

where $\epsilon_{0}=(1-\alpha)$

\section{References}

1. L. Maruster, S. Maruster, Strong Convergence of the Mann Iteration for $\alpha$ - demicontractive Mappings, Math. Comput. Model. 54 (2011) 2486 $-2492$

2. W. Mann, Mean Value Methods in Iteration, Proc. Amer. Math. Soc. 4 (1953), $506-510$

3. H. L. Hicks, J. D. Kubicek, On the Mann Iteration in Hilbert Spaces, J. Math. Anal. Appl. 59 (1977) 498 - 505

4. A. Rafiq, On the Mann Iteration in Hilbert Spaces, Nonlinear Analysis 66 (2007) $2230-2236$

5. St. Maruster, The Solution by Iteration of Nonlinear Equations in Hilbert Spaces, Proc. Amer. Math. Soc. 63 (1)(1977) 767 - 773

6. C. E. Chidume, St. Maruster, Iterative Methods for the Computation of Fixed points of Demicontractive Mappings, J. Comput. Appl. Math. 234(3)(2010) 861 - 882 
7. St. Maruster, Sur le Calcul des Zeros d'un Operateur Discontinu par Iteration, Canad. Math. Bull. 16(4)(1973) 541 - 544

8. M. O. Osilike, Strong and Weak Convergence of the Ishikawa Iteration Method for a Class of Nonlinear Equations, Bull. Korean Math. Soc. $37(1)(2000) 153-169$

9. C. E. Chidume, The Solution by Iteration of Nonlinear Equations in Certain Banach Spaces, J. Nigerian Math. Soc.3(1984) 57 - 62

Received: September 1, 2013 